\title{
New Exact Solutions of the Time-Fractional Nonlinear Dispersive KdV Equation
}

\author{
Yusuf Pandir, Yusuf Gurefe, and Emine Misirli
}

\begin{abstract}
In this study, new version of the extended trial equation method is applied the nonlinear fractional partial differential equations. The fractional partial differential equations can be turned into the nonlinear non-fractional ordinary differential equations by the fractional derivative and traveling wave transformation. So, we find some traveling wave solutions to the time-fractional nonlinear dispersive $\mathrm{KdV}$ equation by the using of the complete discrimination system for polynomial method. As a result, these exact solutions to this nonlinear problem are constructed such as single king solution and hyperbolic function solutions.
\end{abstract}

Index Terms - The extended trial equation method, Time fractional nonlinear dispersive $\mathrm{KdV}$ equation, elliptic integral functions.

\section{INTRODUCTION}

Recently, the fractional differential equations play an important role in applied physics, applied mathematics, chemistry and engineering. The nonlinear fractional partial differential equations represent the mathematical modelling of various real life problems. In order to solve these problems, a general method cannot be defined even in the most useful works. A great deal of work with the approximate solutions for fractional nonlinear partial differential equations is being done [1]-[3]. Also, some new methods for the exact solutions of the nonlinear fractional differential equations have been constructed. The exact solutions of these problems, when they exist, are very important in the understanding of the nonlinear fractional physical phenomena. There are a lot of methods which can be constituted the wave solutions for some time fractional differential equations [4], [5]. Single kink soliton solutions, compacton-like solutions, singular solitons and other solutions have been found by use of these approaches. Apart from all these, some new exact solutions are obtained by using the trial equation methods. Some of them are elliptic integral $F, E$ and $\Pi$ function solutions.

In Section II, we provide at some of the definitions and properties of fractional analysis and also give a new version of the extended trial equation method for time-fractional nonlinear equations. The main feature of this method can be applied to the different equations.

In Section III, as an application, we solve the nonlinear

Manuscript received February 1, 2013; revised June 27, 2013. This work was supported by Yozgat University Foundation.

Yusuf Pandir and Yusuf Gurefe are with Mathematics Dept., Bozok University, Yozgat, 66100 Turkey (e-mail: yusufpandir@ gmail.com, ygurefe@gmail.com).

Emine Misirli is with Mathematics Dept., Ege University, Bornova-İzmir, 35040 Turkey (e-mail: emine.misirli@ ege.edu.tr). fractional partial differential equation such as the time-fractional nonlinear dispersive $\mathrm{KdV}$ equation [6, 7]

$$
\frac{\partial^{\alpha} u}{\partial t^{\alpha}}+a\left(u^{2}\right)_{x}+\left(u(u)_{x x}\right)_{x}+\left(u(u)_{x}\right)_{x x}=0
$$

where $a$ is real valued constant, $\left(u(u)_{x x}\right)_{x}$ and $\left(u(u)_{x}\right)_{x x}$ are dispersive terms. $\frac{\partial^{\alpha}}{\partial t^{\alpha}}$ is Jumarie's modified Riemann-Liouville derivative of order $\alpha$ defined in Section 2. Eq. (1) governs the behavior of weakly nonlinear ion acoustic waves in a plasma comprising cold ions and hot isothermal electrons in the presence of a uniform magnetic field. Early studies for the classical nonlinear dispersive $\mathrm{KdV}$ equation have been established some profound results and it seems that detailed studies of the nonlinear fractional differential equation are only beginning. Using the new version of the extended trial equation method, we find some new exact solutions of the time-fractional nonlinear physical problem. The purpose of this paper is to obtain exact solutions of the time-fractional nonlinear dispersive $\mathrm{KdV}$ equation by new version of extended trial equation method, and to determine the accuracy of this method in solving these kinds of problems.

\section{PRELIMINARIES}

In this part of the paper, it would be helpful to give some definitions and properties of the fractional calculus theory. For an introduction to the classical fractional calculus we indicate the reader to [1]-[3]. Here, we shortly review the modified Riemann-Liouville derivative from the recent fractional calculus proposed by Jumarie [8]-[10]. Let $\mathrm{f}:[0,1] \rightarrow \mathfrak{R}$ be a continuous function and $\alpha \in(0,1)$. The Jumarie modified fractional derivative of order $\alpha$ and $f$ may be defined by expression of [11] as follows:

$D_{x}^{\alpha} f(x)= \begin{cases}\frac{1}{\Gamma(-\alpha)} \int_{0}^{x}(x-\xi)^{-\alpha-1}[f(\xi)-f(0)] d \xi, & \alpha<0 ; \\ \frac{1}{\Gamma(1-\alpha)} \frac{d}{d x} \int_{0}^{x}(x-\xi)^{-\alpha}[f(\xi)-f(0)] d \xi & 0<\alpha<1 ; \\ \left(f^{(n)}(\xi)\right)^{\alpha-n} & , n \leq \alpha \leq n+1, n \geq 1\end{cases}$

In addition to this statement, fractional modified Riemann-Liouville derivative are summarized below which are commonly used features in this paper. Some of the useful formulas are given as follows: 


$$
\begin{aligned}
& D_{x}^{\alpha} k=0 \\
& D_{x}^{\alpha} x^{\mu}= \begin{cases}0, & \mu \leq \alpha-1 \\
\frac{\Gamma(\mu+1)}{\Gamma(\mu-\alpha+1)} x^{\mu-x}, & \mu>\alpha-1\end{cases} \\
& D_{x}^{\alpha}(u(x) v(x))=v(x) D_{x}^{\alpha} u(x)+u(x) D_{x}^{\alpha} v(x)
\end{aligned}
$$

Some new trial equation methods were defined in literature [12]-[24]. In this paper, a new approach to the extended trial equation method will be given. In order to apply this method to fractional nonlinear partial differential equations, we consider the following steps.

Step 1. We consider time fractional partial differential equation in two variables and a dependent variable $u$

$$
P\left(u, D_{t}^{\alpha} u, u_{x}, u_{x x}, u_{x x x}, \cdots\right)=0
$$

and take the wave transformation

$$
u(x, t)=u(\eta), \quad \eta=k x-\frac{\lambda t^{\alpha}}{\Gamma(1+\alpha)}
$$

where $\lambda \neq 0$. Substituting Eq. (5) into Eq. (4) yields a nonlinear ordinary differential equation

$$
N\left(u, u^{\prime}, u^{\prime \prime}, u^{\prime \prime \prime}, \cdots\right)=0
$$

Step 2. Take trial equation as follows:

$$
\left(u^{\prime}\right)^{2}=\frac{F(u)}{G(u)}=\frac{\sum_{i=0}^{n} a_{i} u^{i}}{\sum_{j=0}^{l} b_{j} u^{j}}
$$

and

$$
u^{\prime \prime}=\frac{F^{\prime}(u) G(u)-F(u) G^{\prime}(u)}{2 G^{2}(u)}
$$

where $F(u)$ and $G(u)$ are polynomials. Substituting above relations into Eq. (6) yields an equation of polynomial $\Omega(u)$ of $u$ :

$$
\Omega(u)=\rho_{s} u^{s}+\cdots+\rho_{1} u+\rho_{0}=0
$$

According to the balance principle, we can get a relation of $n$ and $l$. We can compute some values of $n$ and $l$.

Step 3. Let the coefficients of $\Omega(u)$ all be zero will yield an algebraic equations system:

$$
\rho_{i}=0, \quad i=0, \cdots, s
$$

Solving this system, we will specify the values of $a_{0}, \cdots, a_{n}$ and $b_{0}, \cdots, b_{l}$.

Step 4. Reduce Eq. (7) to the elementary integral form

$$
\pm\left(\mu-\mu_{0}\right)=\int \sqrt{\frac{G(u)}{F(u)}} d u
$$

Using a complete discrimination system for polynomial to classify the roots of $F(u)$, we solve Eq. (11) with the help of MATHEMATICA and classify the exact solutions to Eq. (6). In addition, we can write the exact traveling wave solutions to Eq. (4), respectively.

\section{APPLiCATION to THE FRACTIONAL GENERALIZED KDV EQUATION}

In the case of $\alpha=1, \mathrm{Eq}$. (1) reduces to the classical nonlinear dispersive $\mathrm{KdV}$ equation. Many researchers have tried to get the exact solutions of this equation by using a variety of methods [25]. Compactons, solitary patterns, periodic and solitary traveling plane waves solutions of this equation are found.

Let us consider the travelling wave solutions of Eq. (1), and we perform the transformation $u(x, t)=u(\eta)$, $\eta=k x-\frac{\lambda t^{\alpha}}{\Gamma(1+\alpha)}$ where $k, \lambda$ are constants. Then, integrating this equation with respect to $\eta$ and setting the integration constant to zero, we get

$$
-\lambda u+a k u^{2}+k^{3}\left(u^{\prime}\right)^{2}+2 k^{3} u u^{\prime \prime}=0
$$

Substituting, Eqs. (7) and (8) into Eq. (12) and using balance principle yields

$$
n=l+2
$$

This resolution procedure is applied and we obtain results as follows:

\section{Case 1:}

If we take $l=0$ and $n=2$, then

$$
\left(u^{\prime}\right)^{2}=\frac{a_{0}+a_{1} u+a_{2} u^{2}}{b_{0}}, \quad u^{\prime \prime}=\frac{a_{1}+2 a_{2} u}{2 b_{0}},
$$

where $a_{2} \neq 0, b_{0} \neq 0$. Thus, we have a system of algebraic equations from the coefficients of polynomial of $u$. Solving the algebraic equation system (10), we get

$$
a_{0}=0, a_{1}=a_{1}, a_{2}=-\frac{a b_{0}}{3 k}, b_{0}=b_{0}, \lambda=\frac{2 k^{3} a_{1}}{b_{0}}
$$

Substituting these coefficients into Eq. (11), we have

$$
\pm\left(\mu-\mu_{0}\right)=\sqrt{-\frac{3 k}{a}} \int \frac{d u}{\sqrt{u^{2}-\frac{3 k a_{1}}{a b_{0}} u}}
$$

Integrating eq. (15), we procure the solutions to the Eq. (1) as follows:

$$
\begin{gathered}
\pm\left(\mu-\mu_{0}\right)=A \ln \left|u-\alpha_{1}\right| \\
\pm\left(\mu-\mu_{0}\right)=2 A \ln \left|\sqrt{u-\alpha_{1}}+\sqrt{u-\alpha_{2}}\right|
\end{gathered}
$$

where $A=\sqrt{-\frac{3 k}{a}}$. Also $\alpha_{1}, \alpha_{2}$ are the roots of the polynomial equation

$$
\mathrm{u}^{2}+\frac{\mathrm{a}_{1}}{\mathrm{a}_{2}} \mathrm{u}+\frac{\mathrm{a}_{0}}{\mathrm{a}_{2}}=0
$$

Therefore, we find solutions

$$
u(x, t)=\alpha_{1}+\exp \left[\frac{1}{A}\left(k x-\frac{2 k^{3} a_{1} t^{\alpha}}{b_{0} \Gamma(1+\alpha)}-\eta_{0}\right)\right]
$$




$$
\begin{aligned}
\mathrm{u}(\mathrm{x}, \mathrm{t})= & \frac{2\left(\alpha_{1}+\alpha_{2}\right)+\left(\alpha_{1}-\alpha_{2}\right)^{2} \exp \left[\frac{1}{\mathrm{~A}}\left(\mathrm{kx}-\frac{2 \mathrm{k}^{3} \mathrm{a}_{1} \mathrm{t}^{\alpha}}{\mathrm{b}_{0} \Gamma(1+\alpha)}-\eta_{0}\right)\right]}{4} \\
& \frac{+\exp \left[-\frac{1}{\mathrm{~A}}\left(\mathrm{kx}-\frac{2 \mathrm{k}^{3} \mathrm{a}_{1} \mathrm{t}^{\alpha}}{\mathrm{b}_{0} \Gamma(1+\alpha)}-\eta_{0}\right)\right]}{4}
\end{aligned}
$$

If we take $\eta_{0}=\alpha_{2}=0$ and $\alpha_{1}=1$, then the solutions (19)-(20) can reduce to single king solution and the hyperbolic function solution respectively,

$$
\begin{array}{r}
u(x, t)=1+\exp \left[B\left(x-\lambda_{1} t^{\alpha}\right)\right] \\
u(x, t)=\frac{1}{2}\left(1+\cosh \left[B\left(x-\lambda_{1} t^{\alpha}\right)\right]\right)
\end{array}
$$

where $B=\frac{k}{A}, \lambda_{1}=\frac{2 k^{2} a_{1}}{b_{0} \Gamma(1+\alpha)}$. Here, $B$ is the inverse width of the solitons.

Remark 1. Using the new version of the extended trial equation method we obtained the solutions (21)-(22) and results have been checked by Mathematica. To our knowledge, the hyperbolic function solution and single king solution that we find in this paper are not shown in the previous literature. These results are new traveling wave solutions of Eq. (1).
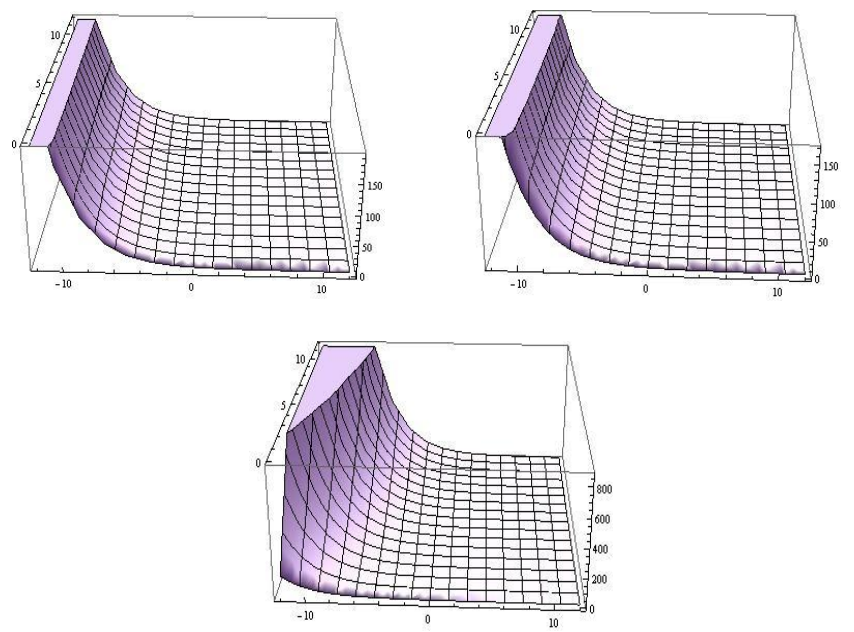

Fig. 1. Graph of the solution (21) corresponding to the values $\alpha=0.01,0.025$ and $\alpha=0.5$ from left to right when

$$
k=-0.5, a=1, a_{1}=2.5 \text { and } b_{0}=0.5 \text {. }
$$

\section{Case 2:}

If we take $l=1$ and $n=3$, then

$$
\left(u^{\prime}\right)^{2}=\frac{a_{0}+a_{1} u+a_{2} u^{2}+a_{3} u^{3}}{b_{0}+b_{1} u},
$$

$u^{\prime \prime}=\frac{\left(b_{0}+b_{1} u\right)\left(a_{1}+2 a_{2} u+3 a_{3} u^{2}\right)-b_{1}\left(a_{0}+a_{1} u+a_{2} u^{2}+a_{3} u^{3}\right)}{2\left(b_{0}+b_{1} u\right)^{2}}$,

where $a_{3} \neq 0, b_{1} \neq 0$. Respectively, solving the algebraic equation system (10) yields

$$
\begin{aligned}
& a_{0}=0, \quad a_{1}=-\frac{a b_{0}\left(3 k a_{2}+a b_{0}\right)}{9 a_{3}}, a_{2}=a_{2}, a_{3}=a_{3}, \\
& b_{0}=b_{0}, \quad b_{1}=-\frac{3 k a_{3}}{a}, \lambda=-\frac{2 a k\left(3 k a_{2}+a b_{0}\right)}{9 a_{3}} .
\end{aligned}
$$

Substituting these results into Eq. (11), we have

$$
\pm\left(\mu-\mu_{0}\right)=\sqrt{-\frac{3 k}{a}} \int \frac{d u}{\sqrt{u^{3}+\frac{a_{2}}{a_{3}} u^{2}-\frac{a b_{0}\left(3 k a_{2}+a b_{0}\right)}{9 a_{3}^{2}} u}}
$$

Integrating Eq. (26), we obtain the solutions to the Eq. (1) as follows:

$$
\begin{aligned}
& \pm\left(\mu-\mu_{0}\right)=2 A\left(\ln \left|\sqrt{\frac{b_{0}+b_{1} u}{b_{1}}}+\sqrt{u-\alpha_{1}}\right|-\sqrt{\frac{b_{0}+b_{1} u}{b_{1}\left(u-\alpha_{1}\right)}}\right), \\
& \pm\left(\mu-\mu_{0}\right)=\frac{2 A}{\sqrt{b_{1}\left(\alpha_{2}-\alpha_{1}\right)}}\left\{\begin{array}{l}
\left.\sqrt{b_{0}+b_{1} \alpha_{1}} \arctan \left[\sqrt{\frac{\left(b_{0}+b_{1} \alpha_{1}\right)\left(u-\alpha_{2}\right)}{\left(b_{0}+b_{1} u\right)\left(\alpha_{2}-\alpha_{1}\right)}}\right]\right\}, \\
+\sqrt{\alpha_{2}-\alpha_{1}} \ln \left|\sqrt{\frac{b_{0}+b_{1} u}{b_{1}}}+\sqrt{u-\alpha_{2}}\right|
\end{array}\right) \\
& \pm\left(\mu-\mu_{0}\right)=\frac{2 A}{\sqrt{b_{1}\left(\alpha_{1}-\alpha_{2}\right)\left(b_{0}+b_{1} \alpha_{3}\right)}}\left(\begin{array}{l}
\left(b_{0}+b_{1} \alpha_{1}\right) F(\varphi, l)+ \\
\left(\alpha_{3}-b_{1} \alpha_{1}\right) \Pi(\varphi, n, l)
\end{array}\right),
\end{aligned}
$$

where

$$
F(\varphi, l)=\int_{0}^{\varphi} \frac{d \psi}{\sqrt{1-l^{2} \sin ^{2} \psi}}, \Pi(\varphi, n, l)=\int_{0}^{\varphi} \frac{d \psi}{\left(1+n \sin ^{2} \psi\right) \sqrt{1-l^{2} \sin ^{2} \psi}}
$$

and

$$
\begin{gathered}
\varphi=\arcsin \sqrt{\frac{\left(u-\alpha_{3}\right)\left(\alpha_{2}-\alpha_{1}\right)}{\left(u-\alpha_{1}\right)\left(\alpha_{2}-\alpha_{3}\right)}}, n=\frac{\alpha_{3}-\alpha_{2}}{\alpha_{1}-\alpha_{2}} \\
l^{2}=\frac{\left(b_{0}+b_{1} \alpha_{1}\right)\left(\alpha_{3}-\alpha_{2}\right)}{\left(b_{0}+b_{1} \alpha_{3}\right)\left(\alpha_{1}-\alpha_{2}\right)}
\end{gathered}
$$

Also $\alpha_{1}, \alpha_{2}$ and $\alpha_{3}$ are the roots of the polynomial equation

$$
u^{3}+\frac{a_{2}}{a_{3}} u^{2}+\frac{a_{1}}{a_{3}} u+\frac{a_{0}}{a_{3}}=0
$$
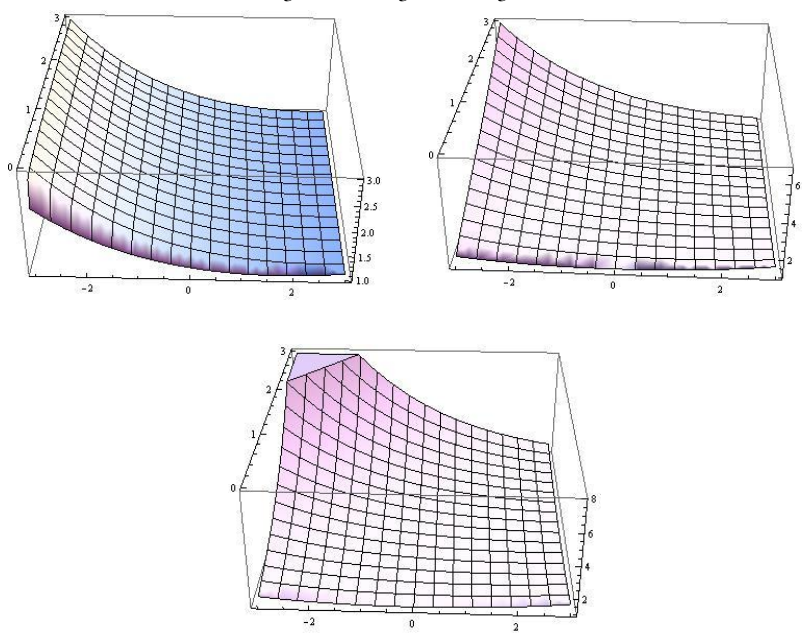

Fig. 2. Graph of the solution (22) corresponding to the values $\alpha=0.01,0.5$ and $\alpha=0.85$ from left to right when $k=-0.5, a=1, a_{1}=2.5$ and $b_{0}=0.5$.
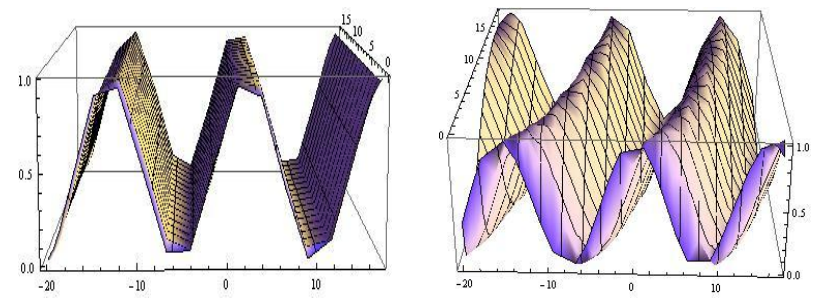


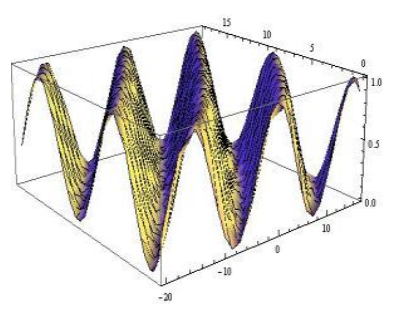

Fig. 3. Graph of the solution (22) corresponding to the values $\alpha=0.01,0.5$ and $\alpha=0.85$ from left to right when $k=-0.5, a=-1, a_{1}=2.5$ and $b_{0}=0.5$

Fig. 1-Fig. 3 show solutions for different values of $\alpha$ when time is fixed. We can see that when the values of $\alpha$ increase, the amplitudes of the solitary pattern solutions also increase.

\section{CONCLUSIONS}

In this paper, new version of the extended trial equation method is studied for the nonlinear time-fractional differential equations. We used it to obtain some soliton and elliptic function solutions to the time-fractional nonlinear dispersive $\mathrm{KdV}$ equation. This method is reliable and effective, and gives several new solution functions such as elliptic integral functions. We think that the proposed method can also be applied to other generalized fractional nonlinear differential equations. In our future studies, we will solve nonlinear fractional partial differential equations by this approach. It is interesting to point out that the fractional derivative parameter $\alpha$ plays an important role in modulating the amplitude of the soliton solution.

\section{REFERENCES}

[1] K. S. Miller and B. Ross, An Introduction to the Fractional Calculus and Fractional Differential Equations, New York, Wiley, 1993,

[2] A. A. Kilbas, H. M. Srivastava, and J. J. Trujillo, Theory and Applications of Fractional Differential Equations, San Diego, Elsevier, 2006.

[3] I. Podlubny, Fractional Differential Equations, San Diego, Academic Press, 1999.

[4] B. Lu, "The first integral method for some time fractional differential equation," Journal of Mathematical Analysis and Applications, vol. 395, pp. 684-693, June 2012.

[5] A. El-Kahlout, T. O. Salim, and S. El-Azab, "Exact solution of time fractional partial differential equation," Applied Mathematical Sciences, vol. 2, no. 52, pp. 2577-2590, 2008.

[6] Z. M. Odibat, "Compact structures in a class of nonlinearly dispersive equations with time-fractional derivatives," Applied Mathematics and Computation, vol. 205, pp. 273-280, 2008.

[7] S. Guo, L. Mei, Y. Fang, and Z. Qiu, "Compacton and solitary pattern solutions for nonlinear dispersive KdV-type equations involving Jumarie's fractional derivative," Physics Letters A, vol. 376, pp. 158-164, 2012.

[8] G. Jumarie, "Modified Riemann-Liouville derivative and fractional Taylor series of non differentiable functions further results," Computers and Mathematics with Applications, vol. 51, no. 9-10, pp. 1367-1376, 2006.

[9] G. Jumarie, "Fractional Hamilton-Jacobi equation for the optimal control of nonrandom fractional dynamics with fractional cost function," Journal of Computational and Applied Mathematics, vol. 23, no. 1-2, pp. 215-228, 2007.

[10] G. Jumarie, "Table of some basic fractional calculus formulae derived from a modified Riemann-Liouville derivative for non-differentiable functions," Applied Mathematics Letters, vol. 22, no. 3, pp. 378-385, 2009.

[11] Z. Ganji, D. Ganji, A. D. Ganji, and M. Rostamian, "Analytical solution of time-fractional Navier-Stokes equation in polar coordinate by homotopy perturbation method," Numerical Methods for Partial Differential Equations, vol. 26, pp. 117-124, 2010.
[12] C. S. Liu, "Trial equation method and its applications to nonlinear evolution equations," Acta Physica Sinica, vol. 54, pp. 2505-2509, 2005.

[13] C. S. Liu, "Trial equation method for nonlinear evolution equations with rank inhomogeneous: mathematical discussions and applications," Communications in Theoretical Physics, vol. 45, pp. 219-223, 2006.

[14] C. S. Liu, "A new trial equation method and its applications," Communications in Theoretical Physics, vol. 45, pp. 395-397, 2006.

[15] C. S. Liu, "Applications of complete discrimination system for polynomial for classifications of traveling wave solutions to nonlinear differential equations," Computer Physics Communications, vol. 181 pp. 317-324, 2010.

[16] C. Y. Jun, "Classification of traveling wave solutions to the Vakhnenko equations," Computers and Mathematics with Applications, vol. 62, pp. 3987-3996, 2011.

[17] C. Y. Jun, "Classification of traveling wave solutions to the modified form of the Degasperis-Procesi equation," Mathematical and Computer Modelling, vol. 56, pp. 43-48, 2012.

[18] Y. Gurefe, A. Sonmezoglu, and E. Misirli, "Application of the trial equation method for solving some nonlinear evolution equations arising in mathematical physics," Pramana-Journal of Physics, vol. 77, pp. 1023-1029, 2011.

[19] Y. Gurefe, A. Sonmezoglu, and E. Misirli, "Application of an irrational trial equation method to high-dimensional nonlinear evolution equations," Journal of Advanced Mathematical Studies, vol. 5, pp. 41-47, 2012.

[20] Y. Pandir, Y. Gurefe, U, Kadak, and E. Misirli, "Classifications of exact solutions for some nonlinear partial differential equations with generalized evolution," Abstract and Applied Analysis, vol. 2012, no. 2012, pp. 1-16, 2012.

[21] Y. Gurefe, E. Misirli, A. Sonmezoglu, and M. Ekici, "Extended trial equation method to generalized nonlinear partial differential equations," Applied Mathematics and Computation, vol. 219, pp. 5253-5260, 2013.

[22] Y. Pandir, Y. Gurefe, and E. Misirli, "Classification of exact solutions to the generalized Kadomtsev-Petviashvili equation," Physica Scripta, vol. 87, pp. 1-12, 2013.

[23] Y. Pandir, Y. Gurefe, and E. Misirli, "The extended trial equation method for some time-fractional differential equations," Discrete Dynamics in Nature and Society, vol. 2013, Article ID 491359, pp. 13, 2013.

[24] X. H. Du, "An irrational trial equation method and its applications," Pramana-Journal of Physics, vol. 75, pp. 415-422, 2010.

[25] A. M. Wazwaz, "Compact and non compact solutions for nonlinear dispersive variants of the generalized KdV equation," Applied Mathematics and Computation, vol. 159, pp. 577-588, 2004.

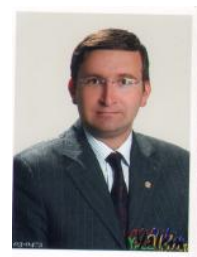

Y. Pandir is an assistant professor in Department of Mathematics at Bozok University; Yozgat (Turkey) $\mathrm{He}$ obtained his M.Sc. degree from Celal Bayar University and Ph.D. degree from Erciyes University. His research interests include fluid mechanics, finite element method, analytical methods for nonlinear differential equations, mathematical physics, and numerical analysis. His research papers are published in Abstract and Applied Analysis, Applied Mathematical Sciences, International Journal of Physical Sciences, Physica Scripta and Journal of Mathematics.

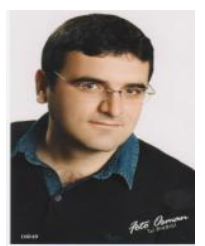

Y. Gurefe is a lecture in Department of Mathematics at Bozok University; Yozgat (Turkey). He obtained his M.Sc. degree from Ege University and Ph.D. degree from Ege University. He has published more than 10 articles in ISI-listed journals. His research interests include multiplicative calculus, analytical and numerical solutions of the linear or nonlinear partial differential equations, nonlinear sciences, mathematical physics.

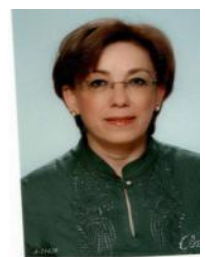

E. Misirli is currently a professor of mathematics in Ege University. She has published more than 20 articles in ISI-listed journals. Her areas of interest are inverse problems, multiplicative calculus, numerical solutions of the partial differential equations, nonlinear sciences. 